Research Article

\title{
Transcriptome sequencing and expression profiling of genes involved in the response to abiotic stress in Medicago ruthenica
}

\author{
Yongjun Shu ${ }^{1}$, Wei $\mathrm{Li}^{1}$, Jinyue Zhao ${ }^{1}$, Ying Liu ${ }^{1}$ and Changhong Guo ${ }^{1}$ \\ ${ }^{1}$ College of Life Science and Technology, Harbin Normal University, Harbin Heilongjiang, China.
}

\begin{abstract}
Medicago ruthenica is a perennial forage legume with the remarkable ability to survive under unfavorable environmental conditions. It has been identified as an excellent species of Medicago that can adapt to various environmental stresses including low temperature, drought, and salinity. To investigate its potential as a genetic resource, we performed transcriptome sequencing and analysis in $M$. ruthenica under abiotic stresses. We generated $>120$ million reads from six cDNA libraries, resulting in 79,249 unique transcripts, most of which were highly similar to transcripts from $M$. truncatula $(44,608,56.3 \%)$ and alfalfa ( $M$. sativa, 48,023, 60.6\%). Based on gene expression profiles, 2,721 transcripts were identified as abiotic stress responsive genes which were predicted to be mainly involved in phytohormone signaling pathways, transcriptional regulation, and ROS-scavenging. These results suggest that they play critical roles in the response to abiotic stress. In summary, we identified genes in our transcriptome dataset involved in the regulation of the abiotic stress response in $M$. ruthenica which will provide a valuable resource for the future identification and functional analysis of candidate genes for adaption to unfavorable conditions. The genes identified here could be also useful for improving stress tolerance traits in alfalfa through molecular breeding in the future.
\end{abstract}

Keywords: Medicago ruthenica; RNA-Seq; transcription factor (TF); abiotic stress; reactive oxygen species (ROS).

Received: October 9, 2017; Accepted: January 3, 2018.

\section{Introduction}

In the natural environment, plant growth is often negatively affected by various unfavorable environmental conditions, such as temperature extremes, drought, and soil salinity. Unfavorable environments have selected for plants that can extensively modify their physiological and biochemical status to adapt to abiotic stresses, and these processes are controlled by complex regulatory networks involving numerous genes (Chinnusamy et al., 2004). Based on their roles in response to stress, the genes are classified into two major functional groups: the first group is mainly comprised of genes encoding osmoprotectants, heat shock proteins (HSP), late embryogenesis abundant proteins (LEA) (Cuevas-Velazquez et al., 2014), transporters (Dos Reis et al., 2012), antioxidants (such as peroxidase, superoxide dismutase, and glutathione peroxidase) (Gill and Tuteja, 2010) and various kinds of metabolism-related proteins. These functional genes play important roles in protecting plants from the effects of environment stress. The second group is made up of genes involved in signal

Send correspondence to Yongjun Shu and Changhong Guo. College of Life Science and Technology, Harbin Normal University, No. 1 of Shida Road, 150025, Harbin, Heilongjiang, China. E-mail: syjun2003@126.com (Yongjun Shu), kaku_2008@163.com (Changhong Guo). transduction and transcriptional regulation, a good example being transcription factors (TFs) that function by regulating the expression or status of other genes. At present, numerous TFs, such as members of the AP2/ERF, MYB, and NAC families, have been identified and characterized as being important regulators in the abiotic stress response in model plants (Golldack et al., 2011; Chen et al., 2012; Mizoi et al., 2012).

Medicago ruthenica L. is an allogamous, diploid $(2 n=16)$ perennial legume forage crop that is widely distributed in Siberia, Mongolia, and northern China. Because of its high tolerance to various extreme environmental conditions, including low temperatures (cold and freezing), drought, and salinity (Campbell et al., 1997; 1999), Balabaev (1934) noted that $M$. ruthenica has excellent prospects as a new forage species, and Campbell et al. (1999) positively evaluated its potential application in low input systems. Wang et al. (2008) hybridized M. ruthenica with $M$. sativa, and produced a new alfalfa cultivar $(M$. sativa cv. 'Longmu No.3') with high tolerance to freezing stress, which suggested that $M$. ruthenica can provide valuable genes for the genetic improvement of alfalfa through breeding. Both Yang et al. (2011) and Guan et al. (2009) investigated the physiological and biochemical responses of $M$. ruthenica to abiotic stresses, and their results have shown that this species is able to regulate its photosynthetic 
rate, stomatal conductance, and $\mathrm{CO}_{2}$ concentration to improve its tolerance to various stresses. However, $M$. ruthenica has received less attention in genetic research than did other Medicago species, such as M. truncatula, $M$. falcata, and M. sativa (alfalfa) (Pennycooke et al., 2008), especially with respect to the molecular mechanisms underlying its tolerance to environmental stress.

Functional gene discovery and prediction have been mainly based on the sequencing of cloned single genes and EST sequencing, which are expensive and low throughput methods. Also, genome-wide gene expression levels are captured by microarray chip hybridization experiments, which are processed using an organism's genetic information, and thus are limited in nonmodel organisms without gene sequences available for chip production. In the last decade, developments in next-generation sequencing (NGS) technologies have facilitated whole transcriptome sequencing, also known as RNA-Seq, which is widely used for revealing complex gene expression patterns in various organisms, including yeast, humans, Arabidopsis, and rice (Wang et al., 2009; Ozsolak and Milos 2011). Recently, RNA-Seq has been increasingly used to identify and characterize genes that control important traits in livestock and crop plants, and it has proven to be a powerful tool for transcriptome analysis, particularly in nonmodel organisms for which a reference genome is not available (Strickler $e t$ al., 2012).

In order to identify important genes that determine resistance to abiotic stress, we performed large-scale RNASeq of $M$. ruthenica under abiotic stresses, and transcriptome profiling allowed us to identify and characterize abiotic stress-responsive genes. The results of our study will provide novel insights into the response to abiotic stresses in M. ruthenica, and will also potentially contribute to the genetic improvement of alfalfa in the future.

\section{Material and Methods}

\section{Plant material and stress treatments}

Seeds of Medicago ruthenica (cv. 'Zhilixing') were the kind gift of Prof. Hong Li from the Heilongjiang Animal Science Institute in Heilongjiang Province, China. This cultivar grows well in Heilongjiang province, with high freezing tolerance. As previously described for $M$. truncatula (Shu et al., 2015, 2016), the seeds of $M$. ruthenica were also germinated in the dark for 24 hours, and the seedlings were then transplanted into pots containing a soil-less mix (perlite and sand, 3:1 by volume). The $M$. ruthenica plants were then grown in a growth chamber (Conviron E15, Canada), and irrigated with 0.5X Hoagland's solution every other day. The growing conditions were set as follows: 14 hour photoperiod, $18 / 24{ }^{\circ} \mathrm{C}$ (light/dark) temperature conditions, and relative humidity ranging from $60-80 \%$. After eight weeks, the seedlings were randomly divided into six groups: (1) for the control group, seedlings were grown at normal conditions as described above; (2) for the cold stress group, the seedlings were transferred to another chamber with the temperature set at $4{ }^{\circ} \mathrm{C}$; (3) for the freezing group, the temperature was $-8{ }^{\circ} \mathrm{C}$; (4) for the osmotic stress group, the seedlings were treated with $300 \mathrm{mM}$ mannitol solution; (5) for the salt stress group, the seedlings were treated with $200 \mathrm{mM} \mathrm{NaCl}$ solution; and (6) for the ABA treatment group, the seedling leaves were sprayed with $100 \mu \mathrm{M}$ ABA solution. All seedlings were harvested $3 \mathrm{~h}$ after treatment. For each treatment group, five whole seedlings were randomly selected and separately bulked, flash frozen in liquid nitrogen, and stored at $-80^{\circ} \mathrm{C}$ prior to use in the experiments.

\section{RNA sequencing library construction and high-throughput sequencing}

Total RNA was extracted from seedlings in the six treatment groups using the RNeasy Plant Mini Kit (Qiagen, Valencia, CA, USA) following the manufacturer's instructions. Total RNAs extracted from the six samples was then quantified using a NanoDrop 2000 spectrophotometer (Thermo Fisher, Waltham, USA) and an Agilent 2100 Bioanalyzer (Agilent Technologies, Santa Clara, USA). The transcriptome sequencing libraries were constructed by BGI-Shenzhen Co. Ltd (Shenzhen, China) as previously described (Shu et al., 2015). Nucleotide sequencing was performed on the Illumina GAII platform, and $100 \mathrm{bp}$ paired-end reads were generated.

\section{De novo assembly and functional annotation}

The raw Illumina sequencing reads were cleaned by removing adapter sequences, PCR duplication reads, empty reads, and reads with low quality scores $(\mathrm{Q}<20)$. The clean reads from the three libraries were then combined, and Trinity software was used for de novo transcriptome assembly with the parameters "min_kmer_cov 2" (Haas et al., 2013). Considering that there is redundancy in the de novo assembly results, we used iAssembler (Zheng et al., 2011) and CD-HIT-EST clusters (Li and Godzik 2006) for further contig assembly. The resulting unique sequences were identified as M. ruthenica unigene transcripts.

To investigate the genetic relationships with $M$. truncatula and M. sativa (alfalfa), we used these transcripts as queries in BLASTN searches against $M$. truncatula (http://www.medicagogenome.org/; Young et al., 2011) and alfalfa transcript (http://plantgrn.noble.org/AGED; O'Rourke et al., 2015) databases with an E-value threshold of 1E-30. The M. ruthenica transcripts, with identities of $>90 \%$, were identified as homologs to M. truncatula or alfalfa transcripts. For functional annotation of the $M$. ruthenica transcripts, we used BLASTP searches against M. truncatula, soybean, and Arabidopsis protein sequences with an E-value of 1E-5, and functional protein annotations, including Gene ontology (GO), and KOG (EuKaryotic Orthologous Groups) annotations, were as- 
signed to the matching $M$. ruthenica transcripts. The GO annotation results were viewed using the online tool WEGO (http://wego.genomics.org.cn) (Ye et al., 2006). To explore regulatory roles of transcription factors in the $M$. ruthenica response to abiotic stresses, these transcripts were analyzed using the iTAK pipeline (http://bioinfo.bti.cornell.edu/tool/itak) (Jin et al., 2014) for plant transcription factor identification and classification, and their respective family members were also evaluated.

\section{Identification of differentially-expressed transcripts}

The clean reads from the six RNA-Seq libraries were mapped to the assembled $M$. ruthenica transcripts using TopHat (Trapnell et al., 2009), and the FPKM values (fragments per kilobase of exon per million fragments mapped) for all transcripts were evaluated with Cufflinks software (Trapnell et al., 2012). Each treatment group was then compared with the control group using $\mathrm{R}$ platform packages, and transcripts with fold changes $\geq 2$ or $\leq 0.5$ and an adjusted $p$-value $\leq 0.05$ were identified as being differentially expressed.

The GO annotation results of these differentially expressed transcripts in the response to abiotic stresses were retrieved, and GO functional enrichment analyses were performed using the topGO package in R (Robinson et al., 2010). The degree of enrichment in each GO term was called the rich factor, which was calculated as follows: [rich factor $=(($ number of transcripts differentially expressed in test GO term)/(number of transcripts differentially expressed with GO annotation $)) /(($ number of transcripts in test GO term)/(number of all transcripts with GO annotation)].

In order to compare genes in the response to abiotic stresses in the Medicago genus, transcriptome data from $M$. truncatula, M. sativa, and M. falcata (Miao et al., 2015) was also downloaded, and we performed RNA-Seq analysis using this data as described above.

\section{Results}

\section{Transcriptome assembly and annotation in $M$. ruthenica}

Using high-throughput Illumina DNA sequencing, $>120$ million reads were generated from six cDNA libraries. Among the raw reads, low quality reads, those containing adapter sequences, or low quality bases were discarded, and the remaining clean reads were deposited in the NCBI SRA database (Accession numbers: SRR4140266, 68-72). These clean reads were then assembled de novo using Trinity software, and redundant transcripts were eliminated using iAssemble and CD-HIT-EST. A total of 79,249 assembled transcripts were generated from the six $M$. ruthenica cDNA libraries, and they were all identified as $M$. ruthenica genes. Mean gene length was 1,020 bp, with
Table 1 - Summary of assembly statistics for the Medicago ruthenica transcriptome.

\begin{tabular}{lc}
\hline Data type & Number \\
\hline Total sequence & 79,249 \\
Number of sequences in 201-500 bp & 32,901 \\
Number of sequences in 500-1000 bp & 16,750 \\
Number of sequences more than $1000 \mathrm{bp}$ & 29,598 \\
Minimal length (bp) & 201 \\
Maximal length (bp) & 1,1741 \\
N50 (bp) & 1,673 \\
Average length (bp) & 1,020 \\
\hline
\end{tabular}

an N50 value of 1,673 bp (details are shown in Table 1). The $M$. ruthenica transcripts had the highest sequence identity with $M$. truncatula and alfalfa, (44,608 and 48,023 transcripts were homologous with $M$. truncatula and alfalfa, respectively; Figure 1). As shown from BLAST search results, 51,115 (64.5\%) of the assembled transcripts were identified with significant hits to combinations of proteins from M. truncatula, soybean, and Arabidopsis.

It is worthy of note that longer unigenes had more hits than did the short ones (Figure 2), implying that a percentage of the unigene annotations was positively correlated with sequence length, which is consistent with previous reports. Among these unigenes, 45,338 (57.2\%) were assigned at least one GO term from the three main domains "biological process", "molecular function", and "cellular component" (Figure 3). Most genes in the biological process category were mainly classified into the groups "cellular process" (GO:0009987), "metabolic process" (GO:0008152), "biological regulation" (GO:0065007), and "response to stimulus" (GO:0050896). In the "molecular function" category, genes were focused on terms including binding (GO:0005488), catalytic activity (GO:0003824), transporter activity (GO:0005215) and transcription regulator activity (GO:0030528). There were also numerous genes present in specific GO terms; for example, antioxidant activity (GO:0016209), which shows that antioxidants play an important role in the response to abiotic stresses, which has also been reported in other plants.

To find genes involved in the transcription regulation process, these genes were scanned using the iTAK pipeline for identifying transcription factors. In total, 1,953 TFs classified in 79 families were identified from the $M$. ruthenica unigenes (Figure 4). The majority of the $M$. ruthenica TFs were found to be members of the MYB, AP2/ERF, bHLH, and WRKY families, which have been previously shown to play important roles in the abiotic stress response in model plant species such as Arabidopsis, rice, and soybean. 

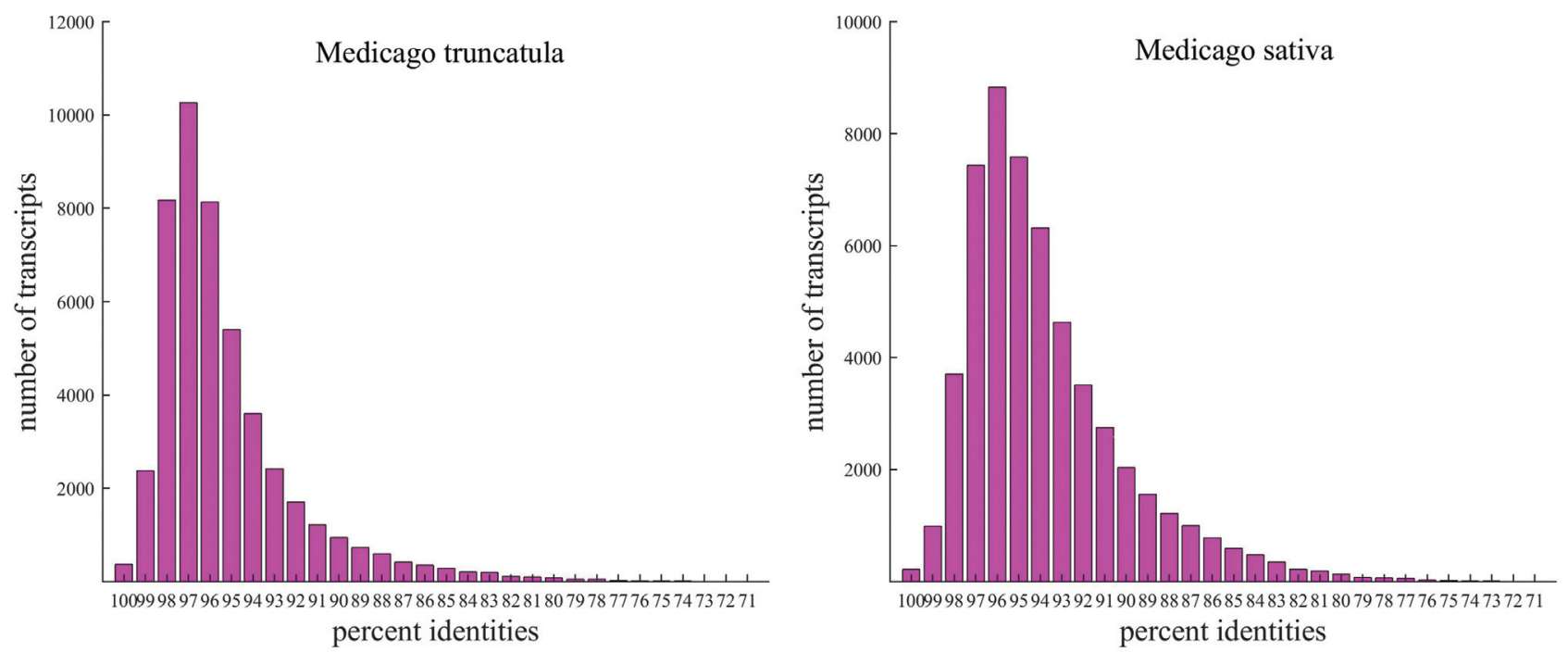

Figure 1 - Sequence identity distributions of unique Medicago ruthenica transcripts against $M$. truncatula and $M$. sativa (alfalfa). The X-axes show the percent similarity of Medicago ruthenica transcripts to the two other Medicago species, and the Y-axes show the numbers of transcripts. The results of this analysis show that transcribed genes from M. ruthenica are highly similar to genes from both M. truncatula and alfalfa.

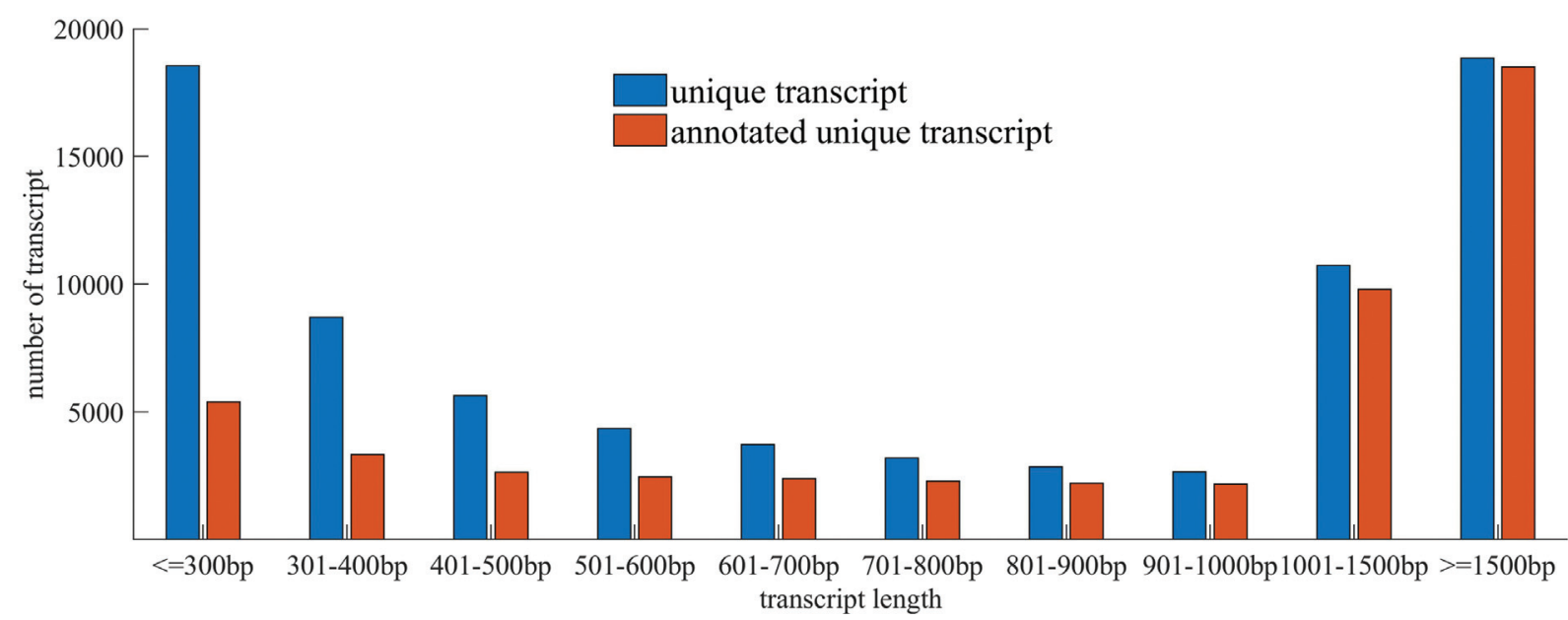

Figure 2 - Length distribution of unique Medicago ruthenica transcripts. Transcript length is shown on the X-axis, and the Y-axis shows the number of transcripts. The numbers and relative proportions of annotated transcripts increase with transcript length, implying that longer transcripts have a higher probability of being annotated.

\section{Identification and characterization of abiotic stress response genes in $M$. ruthenica}

To determine which genes play a role in the abiotic stress response in $M$. ruthenica, five comparisons between the control group and the abiotic stress groups (cold, freezing, osmotic, salt, and ABA), were performed. We identified 2,721 differentially expressed genes with a false discovery rate of 0.05 . Specifically, there were 894 genes that responded to cold stress, 933 to freezing stress, 1,026 to osmotic stress, 913 to salt stress, and 971 to ABA treatment (Figure 5). Among these genes, 33 showed differential expression across all five abiotic stress treatments, implying that these genes are commonly expressed in M. ruthenica in response to abiotic stress, and also that their functions are highly enriched in transcription factors ( $\mathrm{GO}$ analysis results, four genes), indicating that transcriptional regulation is the main means of conferring abiotic stress tolerance. Other genes were specifically either induced or repressed by an individual stress treatment; for example, 345 genes in cold stress, 425 genes in freezing stress, 221 genes in osmotic stress, 263 genes in salt stress, and 152 genes in response to ABA treatment. In addition, a considerable number of genes were simultaneously affected by two stress treatments; for example, 221 genes were differentially expressed in response to both cold and freezing, implying that these two stresses potentially share common regulatory pathways. Similarly, there were 231 genes that responded to both osmotic stress and ABA treatment, and 


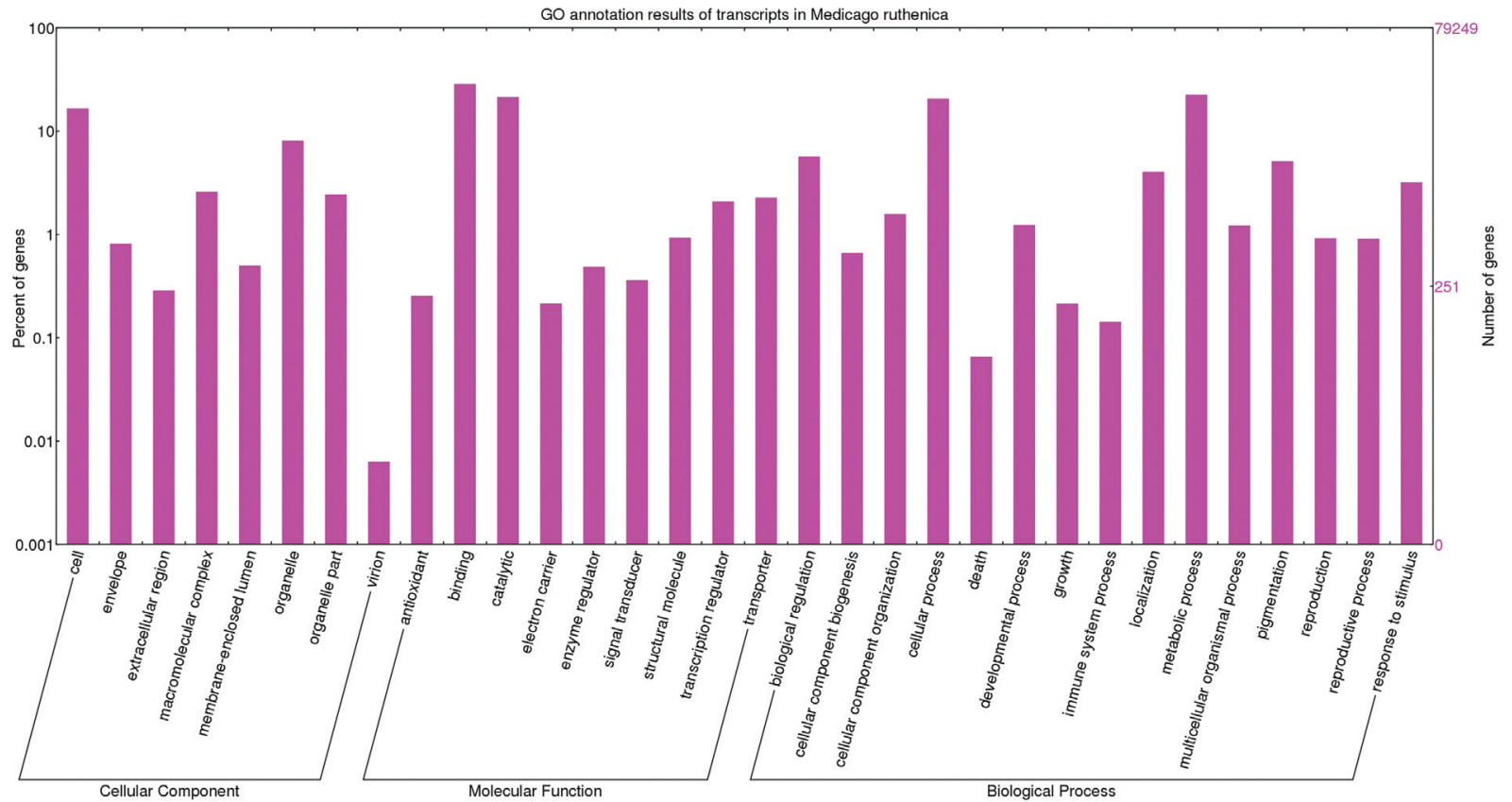

Figure 3 - GO classification results for annotated unique transcripts in Medicago ruthenica. The Medicago ruthenica transcripts were annotated using BLASTP searches against transcriptome databases for M. truncatula, soybean, and Arabidopsis, and the results were categorized and viewed using WEGO. Percentage of genes (y-axis) indicates the proportion of Medicago ruthenica unique transcripts that have relevant GO annotations in the three major GO domains "cellular components", "molecular function", and "biological process".

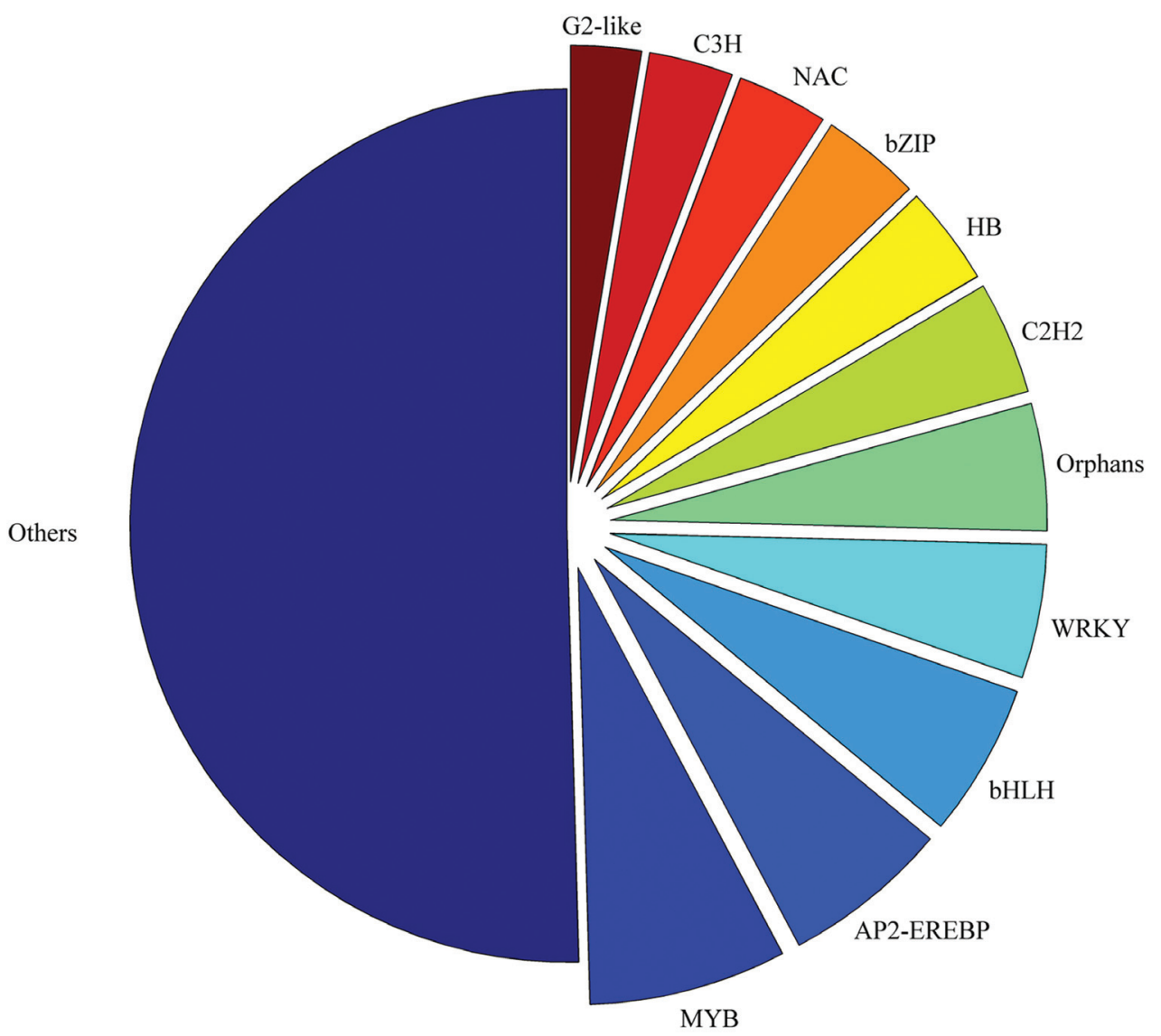

Figure 4 - Distribution of transcription factor genes in the differentially expressed Medicago ruthenica transcriptome sequences. The transcription factor genes were identified and classified using iTAK. The pie plot shows that MYB, AP2/ERF, bHLH, and WRKY are the largest TF family genes expressed in the Medicago ruthenica abiotic stress response. 


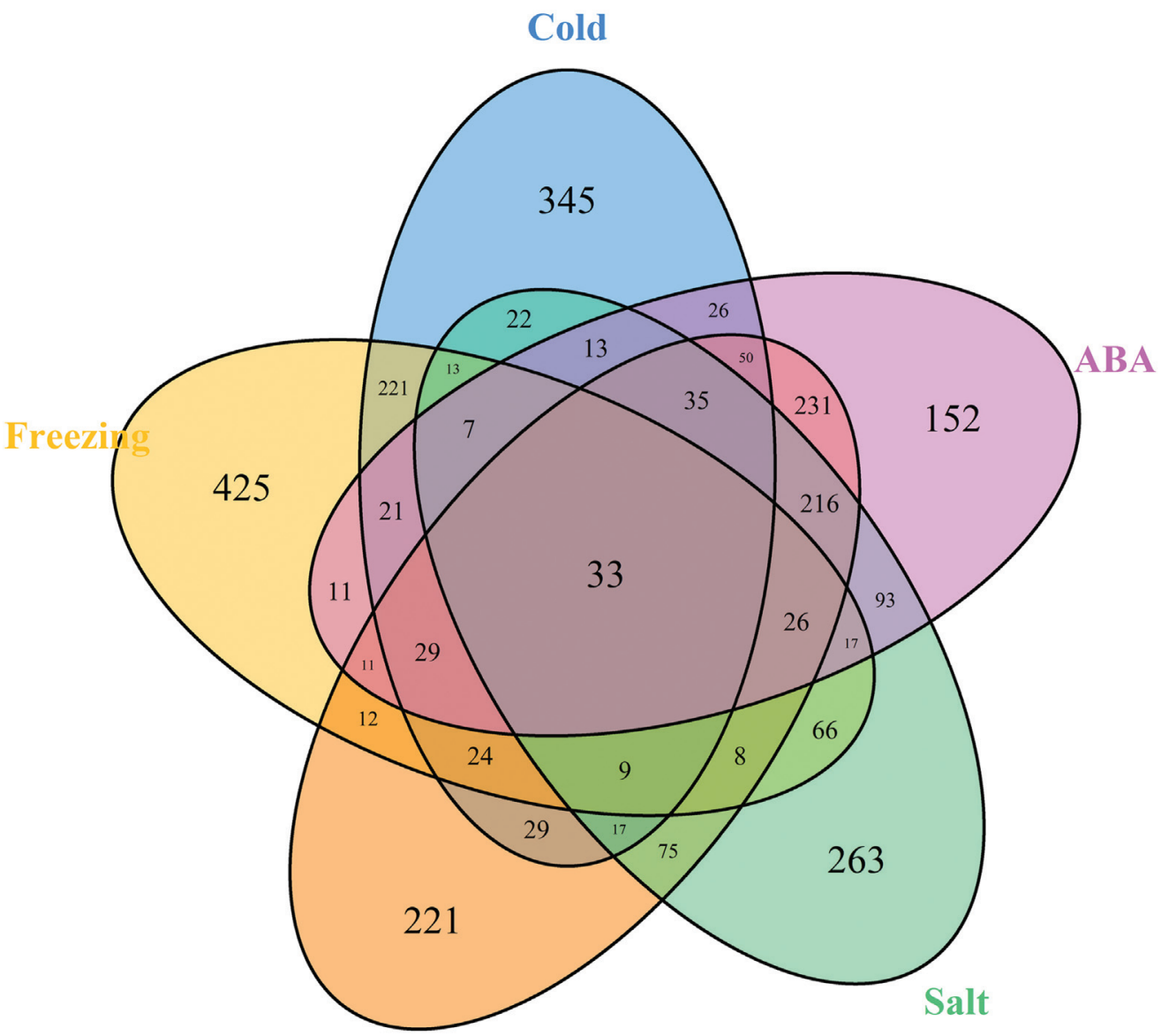

Osmotic

Figure 5 - Diagrammatic representation of the distributions of Medicago ruthenica genes that are differentially expressed in response to five abiotic stresses. The Venn diagram shows the overlap of DEGs in the responses to various abiotic stresses. The cold and freezing stress groups share more commonly expressed genes, while the osmotic, salt and ABA stress groups probably contain other DEGs.

216 that responded to salt and ABA, which were also suggests that ABA plays important roles in the responses to both osmotic and salt stress. To determine their genetic function in the abiotic stress response, $\mathrm{KOG}$ we performed annotation analysis (Figure $\mathrm{S} 1$ ). The results showed that the DEGs involved in abiotic stress could be classified into diverse categories, such as signal transduction, amino acid transport and metabolism, and carbohydrate transport and metabolism, implying that $M$. ruthenica probably employs different genetic regulation pathways to confer tolerance to various abiotic stresses.

\section{Functional annotation and enrichment analysis of the DEGs}

To investigate the functions of these DEGs, we performed GO annotation enrichment analysis using the software package topGO. We found that the DEGs were significantly enriched in $235 \mathrm{GO}$ terms (Table S1). In the "biological processes" category, the main terms, including growth (GO:0040007), immune system process (GO:0002376), photosynthesis (GO:0015979), and response to stimuli (GO:0050896), were highly enriched in the DEGs, suggesting that genes involved in these processes potentially play important roles in the responses to abiotic stresses. In the "molecular function" category, DEGs were significantly enriched in the two terms nucleic acid binding transcription factor activity (GO:0001071) and antioxidant activity (GO:0016209) (Figure 6), implying that TFs and antioxidants have critical functions in the plant response to abiotic stress, which has been shown in other plants. For example, TFs regulate the expression of down-stream functional genes for adapting to stress, while antioxidants protect plant cells from oxidative damage by scavenging reactive oxygen species (ROS). In total, $160 \mathrm{TF}$ genes were identified that responded to one or more specific stress (Figure S2), many of which have been previously shown to have important roles in the abiotic stress response, such as members of the AP2/ERF, bHLH, MYB, WRKY, and NAC TF families (Table S2). Antioxidants, such as peroxidases, ascorbate, and glutathione, eliminate ROS generated by various stresses, protecting plants from intracellular oxidative damage that can occur during stress.

Similar transcriptome analyses were also performed in the model plant M. truncatula, and 3,360 DEGs were 
Enrichment analysis of GO terms response to abiotic stress in Medicago ruthenica

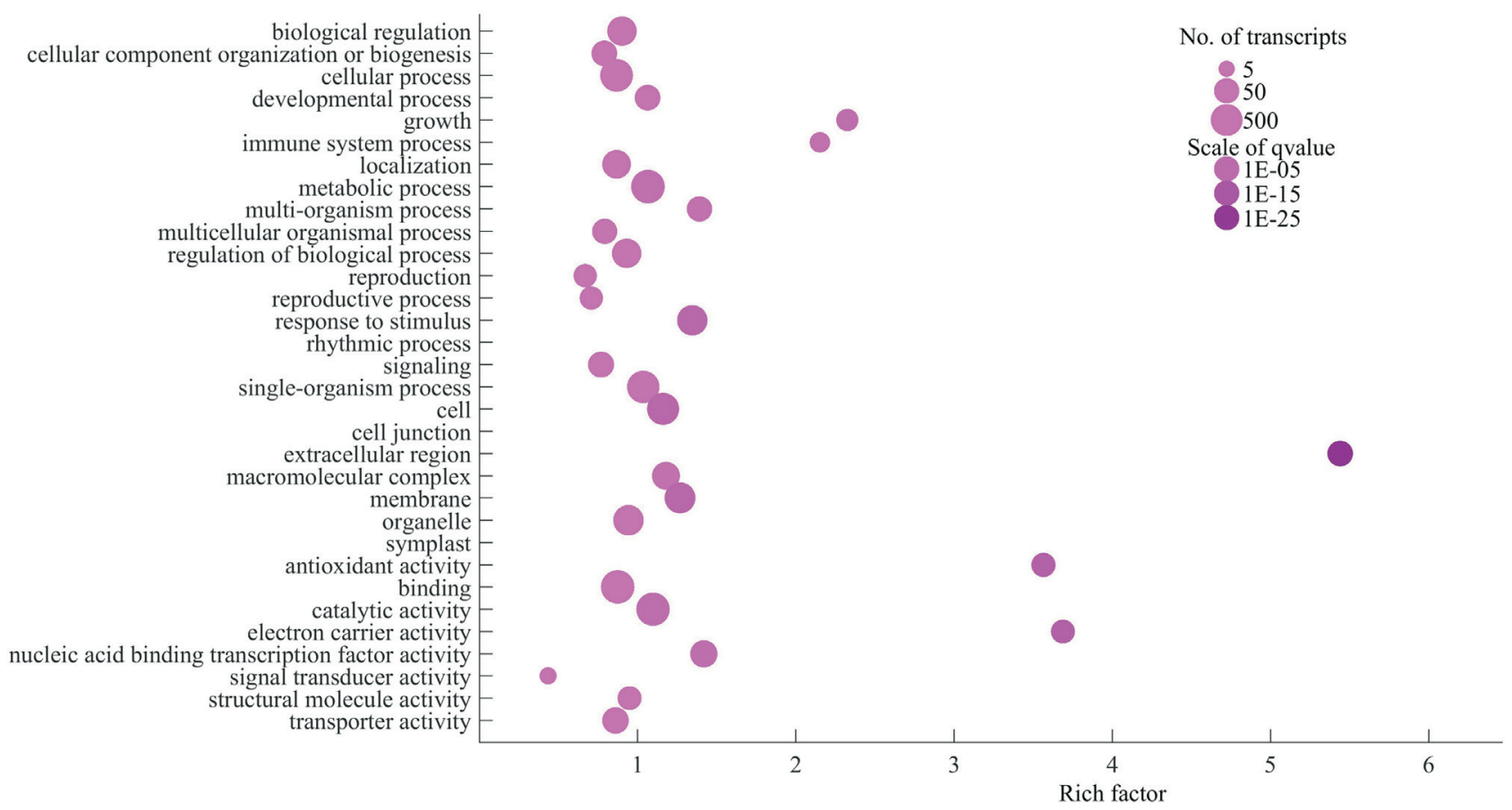

Figure 6 - Gene ontology (GO) enrichment analysis of Medicago ruthenica unique transcripts response to abiotic stress. The enriched transcripts are plotted in the various $\mathrm{GO}$ terms as pink circles, and the sizes of the circles indicate the relative number of transcripts present in each GO term. The color scale is based on $p$-value. Dark pink circles are the most significantly over-represented, while light pink circles represent the least significant terms.

identified (the sequences were downloaded from the NCBI SRA database under accession numbers SRX1056987-92). It is worthy of note that we identified 698 M. ruthenica abiotic stress DEGs in which the homologs were also differentially expressed in M. truncatula (Table S3). We performed 1,000 computer simulations, using 2,721 randomly selected genes from $M$. ruthenica and 3,360 genes from $M$. truncatula in each simulation, and the mean number of genes present in both the M. ruthenica and M. truncatula gene sampling list (homologous gene pairs) was 109, which was much smaller than the number of common genes in the response to abiotic stresses, implying that the expression patterns and functions of many genes are highly conserved between the two species (Figure 7). In addition, functional annotation analysis of these genes showed that they are highly enriched in stress-related processes, including photosynthesis (GO:0015979), oxidation-reduction processes (GO:0055114), and response to stress (GO:0006950) (Table S4).

We also analyzed transcriptome sequences from other Medicago species, including $M$. sativa and $M$. falcata. All DEGs were mapped to the M. truncatula transcript database for multi-species comparison, and the results showed that M. ruthenica and M. falcata are both important resources for the genetic improvement of alfalfa in response to abiotic stress (Figure S3).

\section{Discussion}

In the present study we performed an RNA-Seq analysis of the $M$. ruthenica transcriptome in response to five different abiotic stresses using high-throughput nucleotide sequencing. In total, $>120 \mathrm{M}$ reads were obtained, and 79,249 unigenes were generated by de novo assembly of the pooled RNA-Seq reads. The average length of the assembled unigenes was $1,020 \mathrm{bp}$, and the N50 value was 1,673 $\mathrm{bp}$, indicating that the assembly was of high quality, and implying that many of the unigenes contained full-length coding regions. In addition, $60.6 \%$ of the unigenes $(48,023$ unigenes) had high similarities ( $\geq 90 \%)$ with alfalfa transcripts; considering that it is highly possible that $M$. ruthenica could hybridize with alfalfa, these unigenes could be an important resource for alfalfa genetic improvement, especially with regards to abiotic stress tolerance. The unigenes involved in the response to abiotic stress are discussed below.

\section{Phytohormones that regulate the response to abiotic stress}

Phytohormones, including abscisic acid (ABA), auxins, gibberellins (GA), cytokinins (CK), ethylene (ET), jasmonates (JA), and brassinosteroids (BR), have long been recognized as the key plant hormones that mediate plant growth, development, and responses to abiotic stress (Kumar 2013; Verma et al., 2016). It is now known that 

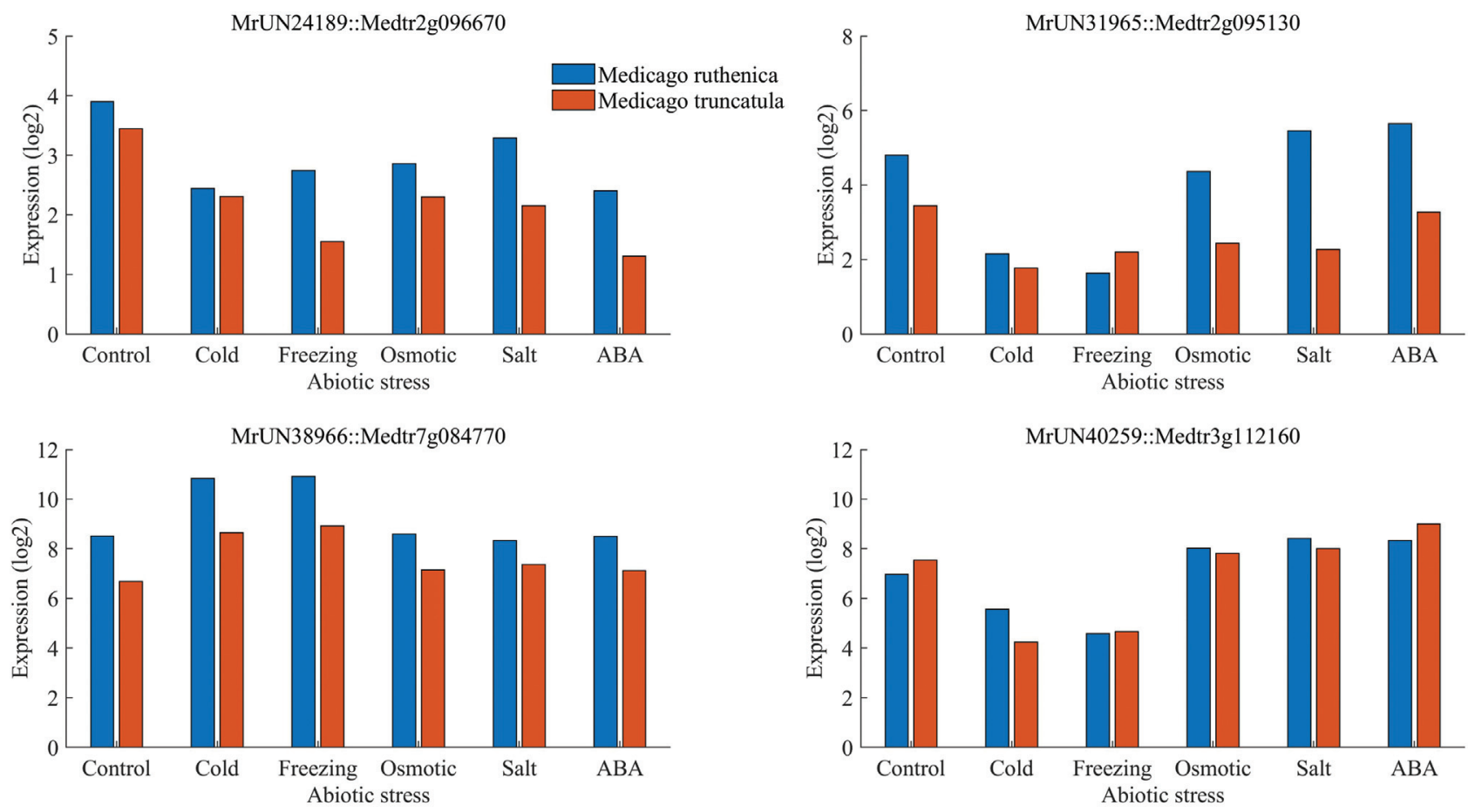

Figure 7 - Comparisons of the expression profiles of four Medicago ruthenica unigenes that are differentially expressed in the response to abiotic stress and their homologs in M. truncatula. Four pairs of homologous genes from Medicago ruthenica and M. truncatula show similar expression profiles in control plants and plants exposed to cold, freezing, osmotic, salt, and ABA stress treatments. The expression levels were calculated using FPKM values.

these phytohormones extensively regulate all aspects of plant stress responses, ranging from signal cascade transduction to modifications in plant developmental processes.

Generally, ABA is the best-studied phytohormone with respect to the plant stress response, and it regulates downstream gene expression through the ABA-responsive element (ABRE) (Sah et al., 2016). Many transcription factors and functional genes have been identified as ABA target genes in various stresses; examples are TFs from the MYB, NAC, and DREB families. In our study, 16 unigenes involved in the response to ABA were identified as being differentially expressed, which supports the important function of ABA in response to abiotic stress (Table S5). Among these regulatory genes, the expression of MrUN03429 and MrUN27218, which both contain a BURP domain, responded to various stresses, which was consistent with previous reports showing the important roles of these genes in abiotic stress ( $\mathrm{Li}$ et al., 2016). GA has been shown to regulate plant growth to withstand stress damage, and we have identified GA-regulated unigenes involved in the cell elongation and division processes, including expansin (MrUN07876), GASA/GAST/Snakin (MrUN19564, MrUN21275, MrUN21674, MrUN27354, and MrUN39273), implying their roles in the stress response via modification of plant growth (Colebrook et al., 2014). Similarly, DEGs were identified and characterized for auxins; several unigenes belonging to the SAUR gene family were identified as being responsive to stress, and these are well known to be regulated by auxins in response to various stresses in many plants (Ren and Gray, 2015).

\section{Transcription factors involved in abiotic stress responses}

Many transcription factors (TFs) have been characterized that play important roles in plant responses to abiotic stresses such as cold, freezing, osmotic stress, and salt. In the current study, we identified $160 \mathrm{TFs}$ that were differentially expressed in the response to abiotic stress in the M. ruthenica transcriptome. Most of these TFs were identified as members of several TF families known to function in plant responses, including AP2/ERF, bHLH, MYB, WRKY, C2H2, and NAC. The largest was the AP2/ERF TF family, members of which have been widely characterized for their roles in cold, osmotic, and salt stress from numerous plants, including Arabidopsis, rice, maize, and soybean. In the present study, we identified 26 AP2/ERF TF genes that showed differential expression in response to abiotic stress, similar to our previous finding in M. truncatula (Shu et al., 2015). This result implies that the critical function of these genes in the abiotic stress response is highly conserved in plants, and that they have a potential application in alfalfa genetics and breeding. In addition, genes from other TF families with important roles in abiotic stress responses have been identified in other plants.

In the MYB family, AtMYB2 has been shown to be induced by dehydration and salt stress (Urao et al., 1996), AtMYB96 was reported to be involved in the response to 
drought stress (Seo et al., 2009), OsMYB3R-2 was characterized and demonstrated to improve freezing, drought, and salt stress tolerance in transgenic plants (Dai et al., 2007), and three soybean MYB genes (GmMYB72, GmMYB96 and GmMYB117) were also shown to be regulated by cold, drought, salt, and and/or ABA stresses (Liao et al., 2008). In our study, were found that 14 MYB TF genes were regulated by various stresses; for example, expression of MrUN10866, MrUN33504, MrUN37588, and MrUN40182 was induced by cold and/or freezing stress, while MrUN28786 was up-regulated by all stresses. These results confirm previous reports in other plants, and confirmed that MYB TFs have positive functions in the response to abiotic stresses. Similar results were confirmed for genes in other well characterized abiotic stress responsive TF families, such as WRKY (Chen et al., 2012) and NAC (Shao et al., 2015).

\section{ROS as key players in abiotic stress responses}

In the plant response to abiotic stress, oxidative stress becomes an important secondary stress due to the accumulation of intracellular reactive oxygen species (ROS), which are harmful for plant growth by destroying cellular components and resulting in programmed cell death (Sewelam et al., 2016). To protect plant seedlings from oxidative damage, plants have established a complex physiological system to scavenge ROS (Baxter et al., 2014; Del Rio and Lopez-Huertas, 2016).

In the present study, we identified numerous genes involved in ROS-scavenging systems that were significantly regulated by abiotic stress based on MapMan annotation results (Usadel et al., 2009). In the M. ruthenica transcriptome, we identified 90 peroxidase (POD) genes, and 17 of these gene were identified as being up- or down-regulated by RNA-Seq, implying that they have a protective function against oxidative damage under abiotic stress conditions. In addition, members of other familiar gene families of ROSscavenging systems have also been shown wo be differentially expressed in response to abiotic stresses (Baxter et al., 2014; Zinta et al., 2016); examples are glutathione (GSHs, two members), glutathione S transferase (GSTs, nine members), peroxiredoxins (PRXs, two members) and glutaredoxins (GRXs, six members). However, the details of ROS-scavenging systems are still largely unknown, and they they are worthy of future study.

\section{Conclusion}

In this study, a transcriptome dataset of genes expressed in $M$. ruthenica in response to five abiotic stresses was generated by high-throughput Illumina RNA sequencing. The RNA-Seq results generated a total of 79,249 assembled transcripts, and 2,721 of these transcripts were identified as abiotic stress responsive genes that are mainly involved in transcriptional regulation, phytohormone signaling pathways, and ROS scavenging. These findings were helpful in exploring the $M$. ruthenica response to abiotic stress, and important genes could be candidates for introduction into alfalfa with a high potential to improve abiotic stress tolerance in the future.

\section{Acknowledgments}

We thank Professor Hong Li (Heilongjiang Animal Science Institute, Heilongjiang Province, China) for kindly providing Medicago ruthenica seeds. We also thank Miss Jun Zhang and Dr. Lili Song (Harbin Normal University) for their helpful technical support in plant growth and treatment. This work was supported by grants from the Natural and Science Foundation of China (31302019 and 31470571), the China Postdoctoral Science Foundation (2016T90307 and 2015M571430), the University Nursing Program for Young Scholar with Creative Talents in Heilongjiang Province (UNPYSCT-2016 to Yongjun Shu), the MOST 863 Project (2013AA102607-5), and the Heilongjiang Province Postdoctoral Science Foundation (LBH-Z14126).

\section{References}

Balabaev GA (1934) Yellow lucernes of Siberia, Medicago ruthenica (L.) Lebd. and M. platycarpos (L.) Lebd.-Bull. App. Bot. Genet. Plant Breed Serv 7:13-123.

Baxter A, Mittler R and Suzuki N (2014) ROS as key players in plant stress signalling. J Exp Bot 65:1229-1240.

Campbell TA, Bao G and Xia ZL (1997) Agronomic evaluation of Medicago ruthenica collected in Inner Mongolia. Crop Sci 37:599-604.

Campbell TA, Bao G and Xia ZL (1999) Completion of the agronomic evaluations of Medicago ruthenica [(L.) Ledebour] germplasm collected in Inner Mongolia. Genet Res Crop Evol 46:477-484.

Chen L, Song Y, Li S, Zhang L, Zhu L and Yu D (2012) The role of WRKY transcription factors in plant abiotic stresses. Biochim Biophys Acta 1819:120-128.

Chinnusamy V, Schumaker K and Zhu JK (2004) Molecular genetic perspectives on cross-talk and specificity in abiotic stress signalling in plants. J Exp Bot 55:225-236.

Colebrook EH, Thomas SG, Phillips AL and Hedden P (2014) The role of gibberellin signalling in plant responses to abiotic stress. J Exp Biol 217:67-75.

Cuevas-Velazquez CL, Rendon-Luna DF and Covarrubias AA (2014) Dissecting the cryoprotection mechanisms for dehydrins. Front Plant Sci 5:583.

Dai X, Xu Y, Ma Q, Xu W, Wang T, Xue Y and Chong K (2007) Overexpression of an R1R2R3 MYB gene, OsMYB3R-2, increases tolerance to freezing, drought, and salt stress in transgenic Arabidopsis. Plant Physiol 143:1739-1751.

Del Rio LA and Lopez-Huertas E (2016) ROS generation in peroxisomes and its role in cell signaling. Plant Cell Physiol 57:1364-1376.

Dos Reis SP, Lima AM and de Souza CR (2012) Recent molecular advances on downstream plant responses to abiotic stress. Int J Mol Sci 13:8628-8647. 
Gill SS and Tuteja N (2010) Reactive oxygen species and antioxidant machinery in abiotic stress tolerance in crop plants. Plant Physiol Biochem 48:909-930.

Golldack D, Luking I and Yang O (2011) Plant tolerance to drought and salinity: Stress regulating transcription factors and their functional significance in the cellular transcriptional network. Plant Cell Rep 30:1383-1391.

Guan B, Zhou D, Zhang H, Tian Y, Japhet W and Wang P (2009) Germination responses of Medicago ruthenica seeds to salinity, alkalinity, and temperature. J Arid Environ 73:135-138.

Haas BJ, Papanicolaou A, Yassour M, Grabherr M, Blood PD, Bowden J, Couger MB, Eccles D, Li B, Lieber M et al. (2013) De novo transcript sequence reconstruction from RNA-Seq using the Trinity platform for reference generation and analysis. Nat Protoc 8:1494-1512.

Jin J, Zhang H, Kong L, Gao G and Luo J (2014) PlantTFDB 3.0: A portal for the functional and evolutionary study of plant transcription factors. Nucleic Acids Res 42: D1182-D1187.

Kumar PP (2013) Regulation of biotic and abiotic stress responses by plant hormones. Plant Cell Rep 32:943.

Li W and Godzik A (2006) Cd-hit: A fast program for clustering and comparing large sets of protein or nucleotide sequences. Bioinformatics 22:1658-1659.

Li Y, Chen X, Chen Z, Cai R, Zhang H and Xiang Y (2016) Identification and expression analysis of BURP domaincontaining genes in Medicago truncatula. Front Plant Sci 7:485.

Liao Y, Zou HF, Wang HW, Zhang WK, Ma B, Zhang JS and Chen SY (2008) Soybean GmMYB76, GmMYB92, and GmMYB177 genes confer stress tolerance in transgenic Arabidopsis plants. Cell Res 18:1047-1060.

Miao Z, Xu W, Li D, Hu X, Liu J, Zhang R, Tong Z, Dong J, Su Z and Zhang L (2015) De novo transcriptome analysis of Medicago falcata reveals novel insights about the mechanisms underlying abiotic stress-responsive pathway. BMC Genomics 16:818.

Mizoi J, Shinozaki K and Yamaguchi-Shinozaki K (2012) AP2/ERF family transcription factors in plant abiotic stress responses. Biochim Biophys Acta 1819:86-96.

O'Rourke JA, Fu F, Bucciarelli B, Yang SS, Samac DA, Lamb JF, Monteros MJ, Graham MA, Gronwald JW, Krom N et al. (2015) The Medicago sativa gene index 1.2: a web-accessible gene expression atlas for investigating expression differences between Medicago sativa subspecies. BMC Genomics 16:502.

Ozsolak F and Milos PM (2011) RNA sequencing: advances, challenges and opportunities. Nat Rev Genet 12:87-98.

Pennycooke JC, Cheng H and Stockinger EJ (2008) Comparative genomic sequence and expression analyses of Medicago truncatula and alfalfa subspecies falcata COLD-ACCLIMATION-SPECIFIC genes. Plant Physiol 146:1242-1254.

Ren H and Gray WM (2015) SAUR proteins as effectors of hormonal and environmental signals in plant growth. Mol Plant 8:1153-1164.

Robinson MD, McCarthy DJ and Smyth GK (2010) edgeR: A Bioconductor package for differential expression analysis of digital gene expression data. Bioinformatics 26:139-140.

Sah SK, Reddy KR and Li J (2016) Abscisic acid and abiotic stress tolerance in crop plants. Front Plant Sci 7:571.
Seo PJ, Xiang F, Qiao M, Park JY, Lee YN, Kim SG, Lee YH, Park WJ and Park C (2009) The MYB96 transcription factor mediates abscisic acid signaling during drought stress response in Arabidopsis. Plant Physiol 151:275-289.

Sewelam N, Kazan K and Schenk PM (2016) Global plant stress signaling: Reactive oxygen species at the cross-road. Front Plant Sci 7:187.

Shao H, Wang H and Tang X (2015) NAC transcription factors in plant multiple abiotic stress responses: progress and prospects. Front Plant Sci 6:902.

Shu Y, Liu Y, Zhang J, Song L and Guo C (2015) Genome-wide analysis of the AP2/ERF superfamily genes and their responses to abiotic stress in Medicago truncatula. Front Plant Sci 6:1247.

Shu Y, Liu Y, Li W, Song L and Guo Z (2016) Genome-wide investigation of microRNAs and their targets in response to freezing stress in Medicago sativa L., based on highthroughput sequencing. G3 (Bethesda) 6:755-765.

Strickler SR, Bombarely A and Mueller LA (2012) Designing a transcriptome next-generation sequencing project for a nonmodel plant species. Am J Bot 99:257-266.

Trapnell C, Pachter L and Salzberg SL (2009) TopHat: Discovering splice junctions with RNA-Seq. Bioinformatics 25:1105-1111.

Trapnell C, Roberts A, Goff L, Pertea G, Kim D, Kelley DR, Pimentel H, Salzberg SL, Rinn JL and Pachter L (2012) Differential gene and transcript expression analysis of RNASeq experiments with TopHat and Cufflinks. Nat Protoc 7:562-578.

Urao T, Noji M, Yamaguchi-Shinozaki K and Shinozaki K (1996) A transcriptional activation domain of ATMYB2, a drought-inducible Arabidopsis Myb-related protein. Plant J 10:1145-1148.

Usadel B, Poree F, Nagel A, Lohse M, Czedik-Eysenberg A and Stitt M (2009) A guide to using MapMan to visualize and compare Omics data in plants: a case study in the crop species, Maize. Plant Cell Environ 9:1211-1229.

Verma V, Ravindran P and Kumar PP (2016) Plant hormonemediated regulation of stress responses. BMC Plant Biol 16:86.

Wang DK, Li H and Luo XY (2008) Crossbreeding of Melilotoides ruthenicus and Medicago sativa. Acta Agrestia Sinica 16:458-465.

Wang Z, Gerstein M and Snyder M (2009) RNA-Seq: A revolutionary tool for transcriptomics. Nat Rev Genet 10:57-63.

Yang JY, Zheng W, Tian Y, Wu Y and Zhou DW (2011) Effects of various mixed salt-alkaline stresses on growth, photosynthesis, and photosynthetic pigment concentrations of Medicago ruthenica seedlings. Photosynthetica 49:275-284.

Ye J, Fang L, Zheng H, Zhang Y, Chen J, Zhang Z, Wang J, Li S, Li R, Bolund L et al. (2006) WEGO: A web tool for plotting GO annotations. Nucleic Acids Res 34:W293-W297.

Young ND, Debelle F, Oldroyd GE, Geurts R, Udvardi MK,Benedito VA, Mayer KF, Gouzy J, Schoof H, Van de Peer Y et al. (2011) The Medicago genome provides insight into the evolution of rhizobial symbioses. Nature 480:520524.

Zheng Y, Zhao L, Gao J and Fei Z (2011) iAssembler: A package for de novo assembly of Roche-454/Sanger transcriptome sequences. BMC Bioinformatics 12:453. 
Zinta G, Khan A, AbdElgawad H, Verma V and Srivastava AK (2016) Unveiling the redox control of plant reproductive development during abiotic stress. Front Plant Sci 7:700.

\section{Supplementary material}

The following online material is available for this article:

Figure S1 - KOG functional classification of Medicago ruthenica differentially expressed transcripts.

Figure S2 - Diagrammatic representation of the distributions of differentially expressed transcripts.

Figure S3 - Heatmap showing the expression profiles of transcription factor genes.
Table S1 - Functional enrichment analysis of GO terms using topGO for differentially expressed genes.

Table S2 - Numbers of DEGs identified as transcription factor genes.

Table S3 - Common DEG pairs identified in both the Medicago ruthenica and Medicago truncatula responses to abiotic stress.

Table S4 - Functional enrichment analysis of GO terms. Table S5 - Phytohormone-related unigenes that were differentially expressed.

Associate Editor: Houtan Noushmehr

License information: This is an open-access article distributed under the terms of the Creative Commons Attribution License (type CC-BY), which permits unrestricted use, distribution and reproduction in any medium, provided the original article is properly cited. 